\title{
Per Linnell si Ivana Markova : Dialogical Approaches to Trust in Communication (2014). Charlottesville : Information Age Publishing
}

\author{
Andreea Ernst-Vintila ${ }^{1}$
}

Les publications d'Ivana Markova, professeure émérite à I'Université de Stirling et professeure invitée à l'Institute of Social Psychology - London School of Economics, ont formé des générations d'étudiants et de chercheurs à travers le monde.

Cette année, Ivana Markova a publié le quatrième volume de la série dédiée à l'exploration des phénomènes de confiance et méfiance dans la communication et, plus généralement, dans la culture, la société, et la vie quotidienne. L'auteure avait lancé le projet auquel nous devons les quatre volumes, intitulé Representations and Discourses of Trust and Democracy: a Social Psychological Approach, en 2000, avec le soutien du Laboratoire Européen de Psychologie Sociale (LEPS) dirigé par Serge Moscovici à la Maison des Sciences de l'Homme.

Le premier volume de cette série s'intitule Trust and Democratic Transition in PostCommunist Europe (2004). Il a été suivi par deux volumes co-édités avec Alex Gillespie (London School of Economics) : Trust and Distrust : Sociocultural perspectives (2008) et Trust and Conflict: Representation, Culture and Dialogue (2012). Le quatrième volume s'intitule Dialogical Approaches to Trust in Communication (2014) et il a été co-édité par Ivana Markova et Per Linnell (Université de Linköping, Suède).

Préfacé par Jaan Valsiner (Clark University), Dialogical Approaches to Trust in Communication dédie 12 chapitres à l'analyse des phénomènes de confiance à travers la communication et le langage dans une perspective multidisciplinaire qui relie études culturelles, sciences de l'éducation, linguistique, psychologie clinique et développementale et, bien évidemment, psychologie sociale. Les auteurs sont des chercheurs avec une variété d'ancrages culturels, travaillant en Amérique Latine (Brésil ${ }^{2}, \mathrm{Chili}^{3}$ ), en Asie (Inde) et en Europe (France, Suisse, Grande-Bretagne, Norvège, Portugal, Suède). Cette diversité disciplinaire et géographique, plutôt rare dans les ouvrages coordonnés par les psychologues sociaux, mais caractéristique de ceux édités par les étudiants et collaborateurs de Serge Moscovici, envoie déjà le premier signal de la richesse que les lecteurs trouveront dans le livre d'Ivana Markova et Per Linnell.

Le volume comprend trois parties. La première regroupe des recherches sur la morale dans les discours sur la confiance et la méfiance. La deuxième se penche sur les phénomènes de confiance et méfiance dans la communication institutionnelle. Enfin, la troisième partie propose un dialogue entre perspectives théoriques qui se répondent. Proche du regard psychosocial théorisé par Moscovici (1984) (le regard ternaire Ego-Alter-Objet sur les phénomènes psychosociaux), la perspective dialogique défendue par Ivana Markova pose la capacité des individus à penser et communiquer la réalité sociale par rapport ou par contraste à l'Autre. Son hypothèse est que la dialogique définit la pensée et le langage

\footnotetext{
${ }^{1}$ Maître de Conférences en Psicologia à l' Université de Reims Champagne-Ardenne - France.

${ }^{2}$ Lívia Mathias Simão - Universidade de São Paulo.

${ }^{3}$ Carlos Cornejo, Pontifcia Universidad Catolica de Chile.
} 
humains et constitue le cadre où s'expriment et s'expliquent les phénomènes de confiance et méfiance.

Le Chapitre 3, intitulé "On ne peut plus faire confiance à personne. Confiance, identité morale et la réconciliation avec le passé " s'inscrit avec subtilité dans cette perspective et présente un intérêt particulier pour les lecteurs qui s'intéressent aux anciennes dictatures. Signé par Cristian Tileaga (Université de Loughborough), ce chapitre se penche sur la façon dont l'identité morale et les questions de confiance / méfiance sont gérées dans l'espace public dans un cas de confession publique faite par un ancien collaborateur de la police secrète en Roumanie communiste avant 1989 - un processus qu'on rencontre dans tous les pays d'Europe de l'Est après la chute du totalitarisme communiste. Plus précisément, le chapitre fait une analyse socio-communicative de la façon dont la confiance et la méfiance s'expriment dans les jeux de langage et des modes spécifiques qu'ont les acteurs sociaux (l'auteur de la confession et ses commentateurs) pour conceptualiser la confiance et la distinguer des concepts similaires.

L'analyse part de deux prémisses. La première pose la confiance comme phénomène de l'ordre (la confiance en tant que substance de l'ordre moral). La seconde est que, dans les anciens pays communistes totalitaires, les mécanismes de contrôle et de surveillance (les polices secrètes telles que la Securitate en Roumanie, la Stasi en Allemagne de l'Est) ont pu fonctionner et se reproduire s'appuyant sur une profonde altération du contrat social fondé sur la confiance, ressource-même de la cohésion et la solidarité sociale. Dans ces conditions, écrit Tileaga, la transition du totalitarisme à la démocratie a été une période non seulement des transformations sociales, politiques et économiques, mais aussi celle de processus psychosociaux de réévaluation du passé, de renégociation des biographies, des identités, de la mémoire et des relations sociales. Si la confiance décrit «le fait que les groupes et les individus supposent la bonne volonté des autres envers les intérêts communs et la division des connaissances nécessaires à l'utilisation des règles explicites de l'action collective " (Warren, 1999, p. 14), alors ces processus psychosociaux frappants ont eu lieu dans le contexte remarquable où la base-même de la société avait été secouée.

L'analyse socio-communicative proposée par Tileaga des jeux de langage mobilisés par l'auteur la confession (une lettre ouverte à un quotidien national) et les commentateurs de celle-ci (les lecteurs du quotidien) a identifié ceux qui rendent pertinente une certaine formulation de la confiance au détriment d'autres alternatives qui pouvaient exister ( la grammaire du sens commun de la confiance »). Par exemple, la phrase "J'ai parfois rapporté sur mes connaissances et des amis, mais je n'ai jamais trahi, je n'ai pas été un agent provocateur " met en évidence, écrit Tileaga, la renégociation de l'identité morale à travers le contraste "parfois " - " jamais". Ce contraste montre que l'auteur de la confession situe son Soi en opposition avec une identité morale nuisible (celle d'un " traître d'amis "). II redéfinit ensuite son identité et son caractère moral alternatifs en niant d'autres formulations possibles ("agent provocateur »). L'analyse montre que, bien que le terme " confiance " n'apparaît pas explicitement dans la confession, il constitue, au même titre que le souci de se redéfinir l'identité et le caractère moral, une grille de lecture indispensable, en l'absence de laquelle la confession n'aurait aucun sens ni pour son auteur, ni pour le public.

Comment s'exprime la confiance et la méfiance dans les commentaires sur la confession ? Choisir de faire une confession publique et se réconcilier dans l'espace public avec son passé suppose d'engager une communauté interprétative et le souci pour la façon dont les membres de la société s'impliquent vis-à-vis du caractère moral de l'Autre (Tileaga, 
2009). Aussi, choisir l'espace public, plutôt que privé, pour lancer une confession peut être regardé avec suspicion et considéré comme fondé par des arrière-pensées liés y compris à la gestion des aspects moraux. Tileaga défend l'idée que ce qui permet à la fois à l'auteur et aux commentateurs de s'engager dans une relation dialogique et dans la négociation du sens de la confession est la grammaire-même de la confiance et la méfiance : dans ces conditions, il est important non seulement qui et quand on peut juger l'Autre, mais aussi dans quel contexte (le contexte ici est le moment où l'on apprend que l'Autre n'est pas ce qu'il semblait). Les commentaires ne portent pas sur l'action elle-même (la délation), mais bien sur ses implications morales. La question de la confiance s'y pose explicitement: "Comment faire confiance à ...? ", "Vous ne pouvez plus faire confiance à qui que ce soit ", "Nous choisissons nous-mêmes en qui nous avons confiance ", etc. Le chapitre révèle avec précision, dans les commentaires de la confession, le passage de la transgression morale individuelle à celle de la responsabilité politique, encore non dévoilée. "Le portrait moral de la société n'est encore ni fini, ni décidé ", écrit Tileaga.

Ce quatrième volume coordonné par Ivana Markova et Per Linnell, s'adresse, comme les trois premiers, non seulement aux étudiants et aux chercheurs en sciences sociales et humaines, mais aussi aux lecteurs qui veulent comprendre (" sérieusement ", écrivent les coordonateurs) les mécanismes de la confiance en société.

\section{Références}

Moscovici, S. (1984). Psychologie sociale. Paris : Presses Universitaires de France.

Tileaga, C. (2009). « Mea Culpa » : The social production of public disclosure and reconciliation with the past. In A. Galasinska \& M. Krzyzanowski (Eds.) Discourse and Transformation in Central and Eastern Europe (pp. 173-187). London : Palgrave.

Warren, M. E. (1999). Introduction. In M. E. Warren (Ed.). Democracy and Trust (pp. 1-21). Cambridge: Cambridge University Press. 